\title{
BMJ Open Tai Chi postural training for dyskinesia rehabilitation: a study protocol for a randomised controlled trial in convalescent ischaemic stroke patients
}

\author{
Chengyang Jing (1) , ${ }^{1,2}$ Kuangshi Li (D) , ${ }^{2}$ Zongheng Li (D) , ${ }^{2}$ Yiting Sun (D) ,,2 \\ Jiabao Wu (1) , ${ }^{1,2}$ Yingjie Li (D) , ${ }^{1,2}$ Yuyue Li (D) , ${ }^{1,2}$ Li Zhou (D) , ${ }^{2}$ Zhe Zhang (D) , \\ Mingzhi Zhao (D) , ${ }^{2}$ Yong Zhang (iD ${ }^{2}$
}

To cite: Jing C, Li K, Li Z, et al. Tai Chi postural training for dyskinesia rehabilitation: a study protocol for a randomised controlled trial in convalescent ischaemic stroke patients. BMJ Open 2021;11:e046003. doi:10.1136/ bmjopen-2020-046003

- Prepublication history for this paper is available online. To view these files, please visit the journal online (http://dx.doi org/10.1136/bmjopen-2020046003).

$\mathrm{CJ}$ and $\mathrm{KL}$ are joint first authors.

Received 17 0ctober 2020 Revised 10 April 2021 Accepted 27 April 2021

Check for updates

(C) Author(s) (or their employer(s)) 2021. Re-use permitted under CC BY-NC. No commercial re-use. See rights and permissions. Published by BMJ.

${ }^{1}$ First Clinical Medical School, Beijing University of Chinese Medicine, Beijing, China

${ }^{2}$ Department of Rehabilitation, Beijing University of Chinese Medicine Affiliated Dongzhimen Hospital, Beijing, China

Correspondence to Dr Zongheng Li;

lee_zongheng@163.com

\section{ABSTRACT}

Introduction Acute ischaemic stroke (AIS) is not only seriously damaging to the physical and mental health of patients, but also has become a major social public health problem. Effective dyskinesia rehabilitation treatment in convalescence is of great significance for AIS patients' prognosis and quality of life. Tai Chi (TC) shows great potential in improving motor function. This trial aims to evaluate the clinical efficacy of modified TC postural training (TPT), and to explore the related central-peripheral neurotransmitter mechanisms.

Methods/design The proposed study will be a multicentre randomised controlled trial. The trial will randomise 120 eligible AIS patients in a 1:1 ratio to receive TPT or Bobath rehabilitation training. Each training session will last $40 \mathrm{~min}$ and will be implemented once a day and five times per week (from Monday to Friday) in a duration of 4 weeks. After finishing the 4-week treatment, another 3-month follow-up period will be seen. Root mean square generated from the surface electromyogram (SEMG) will be the primary outcome. Other SEMG time-domain parameters and frequency-domain parameters and clinical scales assessment will be the secondary outcomes. Peripheral blood samples will be collected at baseline and at the end of 4-week treatment, which will be used to explore the related therapeutic mechanisms. Intentionto-treat analysis and per-protocol analysis will both be implemented in this trial.

Ethics and dissemination The study has been approved by Ethics Committee of Dongzhimen Hospital Affiliated to Beijing University of Chinese Medicine, being granted approval numbers DZMEC-KY-2020-22. The research results will be disseminated through (open access) peerreviewed publications and presentations at conferences. Trial registration number ChiCTR2000032999.

\section{INTRODUCTION}

Stroke is a type of cerebrovascular disease characterised by high morbidity, high disability rate and high mortality. An authoritative survey revealed that stroke had become the leading cause of disability and the second-leading cause of death around

\section{Strengths and limitations of this study}

- To the best of our knowledge, the study is the first randomised controlled trial evaluating the effect of modified Tai Chi postural training (TPT) for dyskinesia rehabilitation in convalescent ischaemic stroke patients by objective parameters (produced by surface electromyogram).

- Neurotransmitter detection will be helpful to explore the therapeutic mechanism of TPT for rehabilitation on dyskinesia.

- Due to the characters of the intervention, the trial could not be double blind. But the electronic data capture system will contribute to quality control of the trial.

- Changes in the patient's condition and a relatively long follow-up period may increase the patient's drop-out rate.

the world. ${ }^{1}$ According to the epidemiological investigation, the incidence rate of stroke in China is as high as 116-219 per 100, 000 person-years, showing an increasing trend in the past years. ${ }^{2}$ Previous studies showed that there were about 7 million stroke patients in China, among whom about $70 \%$ account as acute ischaemic stroke (AIS) patients. ${ }^{34}$ The mortality rate of hospitalised patients with AIS in China was 9\%-9.6\%. Meanwhile, the mortality/ disability rate was $34.5 \%-37.1 \%$ 3 months after onset. When the stroke onset is 1 year, the mortality rate was $14.4 \%-15$. $4 \%$ while the mortality/disability rate was $33.4 \%-33.8 \% .^{56}$ AIS has gradually become a major social public health problem because it not only seriously impairs motor function of AIS patients but also aggravates their mental anxiety. ${ }^{7}$ Since the motor function level of AIS patients is closely related to the prognosis and quality of life, it is particularly important to 
carry out effective rehabilitation of dyskinesia especially in convalescence.

Currently, novel training methods based on virtual reality (VR) technology have been successively applied to the motor function rehabilitation of AIS patients. A number of small sample studies have shown that technology-assisted VR training can effectively improve stroke patients' motor function, active range of motion and dynamic and static balance. ${ }^{8}$ In terms of motor learning after stroke, some scholars have proposed that implicit motor learning may have additional benefits for stroke patients with cognitive impairment or deficits, ${ }^{10}$ since it relies less on working memory resources. ${ }^{11}$ In addition, different frequency and intensity of training will also affect the final rehabilitation effect. ${ }^{12}$ Although different types of novel technology-assisted rehabilitation training have shown good efficacy, their high cost of equipment, or strict and specific patient population selection make it difficult to popularise. ${ }^{9}$ In Chinese stroke rehabilitation practice, manual therapy based on neurodevelopment treatment is still the mainstream, ${ }^{13}$ among which Bobath therapy is the most representative one. The overall principle of Bobath is to give full play to the initiative of patients under the premise of controlling the abnormal movement mode of patients. Bobath rehabilitation also provides appropriate assistance to patients, completes joint and muscle group training, and maximises recovery of limbs motor function. Although Bobath Concept has been widely used in stroke rehabilitation, ${ }^{1415}$ its clinical effect on AIS patients with severe dyskinesia still has limitations. ${ }^{16} 17$ In brief, the treatment needs to be conducted one-on-one under the guidance of a professional rehabilitation therapist and usually lasts for a period of months to years. This not only challenges the medical level in the region where the patients live, but also increases the economic burden of patients. ${ }^{1}$ Therefore, a simpler and more effective therapy is urgently needed for improving the motor function and reducing disability. Tai Chi (TC), which can complement the deficiencies of modern medical treatment in AIS rehabilitation, shows great potential in improving motor function ${ }^{18} 19$ and has been expected to be a new option.

For more than half a century, the scholars have conducted a great number of researches on TC from the perspectives of medicine, nutrition, psychology and biomechanics. ${ }^{20}$ Among them, the clinical application value of TC has been drawn attention to particularly. ${ }^{21-23}$ Studies have shown that TC is effective in the treatment of fibromyalgia and Parkinson's disease ${ }^{24} 25$ and in reversing the risk factors for stroke in middle-aged and elderly adults. ${ }^{26}$ In a study of TC with Berg Balance Scale and other clinical scales selected as outcome measures, the results revealed that the effect of TC training was significantly better than that of the conventional rehabilitation treatment. ${ }^{27}$ However, the previous studies are mainly focused on the integral TC routine and there is still a lack of clinical efficacy study on single postures after decomposition. Furthermore, TC is still not widely applied to the AIS patients with severe dyskinesia. At the same time, there are few studies on the efficacy mechanism of TC. In recent years, some scholars have completed exploratory studies on the biomechanical mechanism, but not many studies pay attention to the correlation between TC and neurotransmitters. The research on the centralperipheral neurotransmitter mechanism of TC promoting the recovery of motor function in AIS patients is still a blank. This study not only aims to evaluate the clinical efficacy and safety of TC postural training (TPT), but also to explore the association between neurotransmitters and TC for promoting the rehabilitation of motor function in AIS patients. The results are promising to establish a foundation for further development of a new, simple, safe and effective therapy applying in convalescence of AIS.

\section{HYPOTHESES}

We speculate that AIS patients may be more prone to exercise-induced central fatigue, thereby inhibiting the recovery of motor function. The special TPT improve this state and promote the rehabilitation of motor function by reducing central fatigue, which may show better results than traditional Bobath therapy.

\section{OBJECTIVES}

\section{Primary objectives}

The primary objectives of the study are:

1. Clinical efficacy of TPT is verified in promoting the rehabilitation of motor function in AIS patients through advanced objective outcome measures.

2. Advantages of curative effect of TPT is verified in convalescence of AIS by comparing the objective outcome measures.

\section{Secondary objectives}

The secondary objectives of the study are:

1. Finding the evidence of the improvement in exerciseinduced central fatigue in ischaemic stroke patients applying TPT, and exploring the related centralperipheral mechanisms.

2. Analysing the therapeutic targets, and providing evidence-based medical evidences for the application of TPT in dyskinesia rehabilitation of ischaemic stroke patients.

\section{METHODS AND ANALYSIS}

\section{Study design, recruitment and procedure}

This multicentre randomised controlled trial will be conducted at three trial sites in Beijing in mainland China: (1) Dongcheng Campus of Dongzhimen Hospital Affiliated to Beijing University of Chinese Medicine, (2) Tongzhou Campus of Dongzhimen Hospital Affiliated to Beijing University of Chinese Medicine and (3) Pinggu Campus of Beijing Hospital of Traditional Chinese Medicine Affiliated to Capital Medical University. A total of 120 participants meeting the inclusion criteria will 


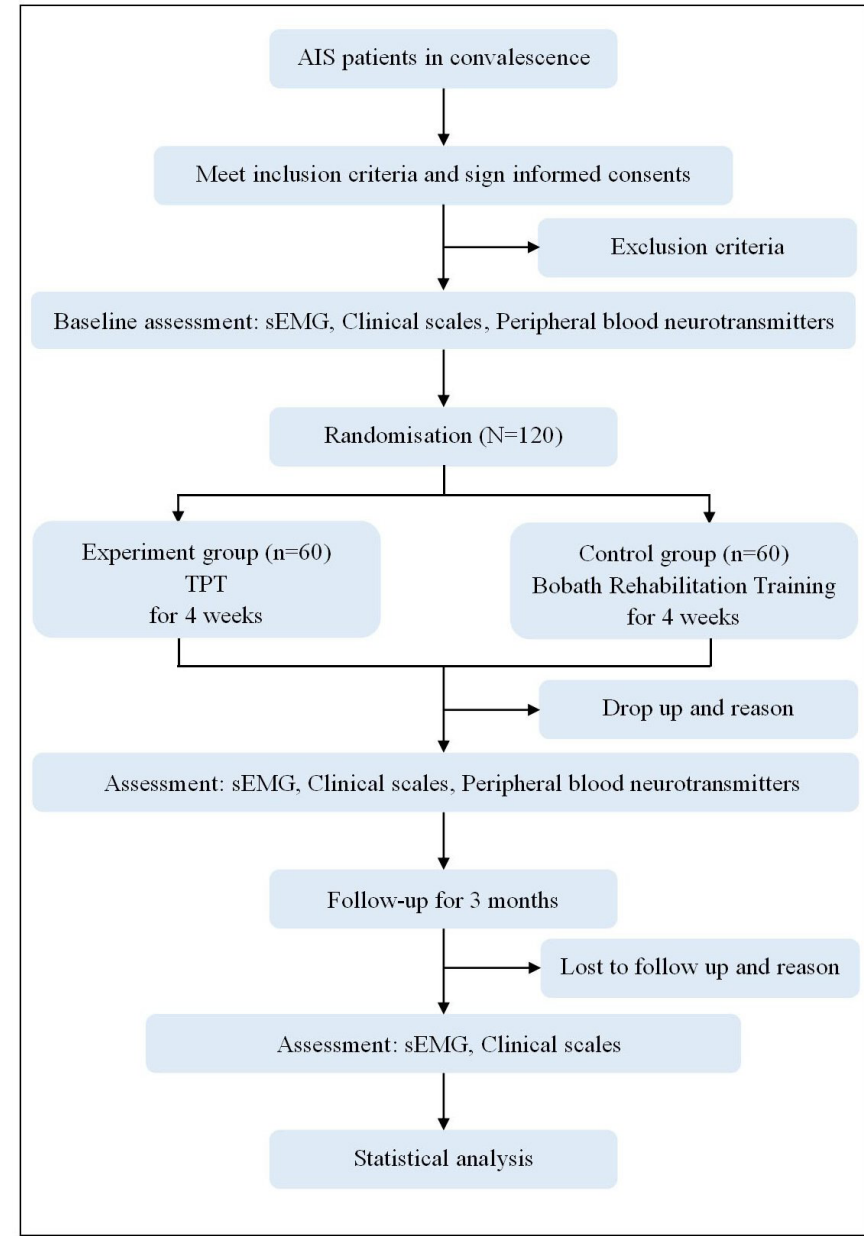

Figure 1 Flow chart of study procedure. AIS, acute ischaemic stroke; sEMG, surface electromyography; TPT, Tai Chi postural training.

be recruited from three trial sites. Patients can obtain specific public recruitment information through posters in the outpatient department or inpatient department. We will communicate with prospective participants about the study details. After the patient voluntarily signs the informed consent, he or she will be invited to participate in the trial. Recruitment will start from 1 June 2020, until when 120 participants are enrolled. The procedure of this trial is shown in figure 1. The schedule of enrolment, intervention and assessments in this trial is shown in table 1 .

\section{Eligibility criteria}

Inclusion criteria

- In accordance with the diagnostic criteria of the 'Chinese guidelines for diagnosis and treatment of AIS' (2018 version), ${ }^{28}$ and confirmed by CT or MRI examination.

- Aged 30-80 years old, male or female, right-handed habit.

- First episode of stroke or with a history of stroke but with no serious neurological dysfunction and modified Ranking Scale grade $\leq 2$.
- AIS patients who are in the subacute phase (from 2 weeks to less than 3 months after onset).

- With dyskinesia but muscle strength $\geq$ III grade (including III grade).

- With symptom of lower limb hemiplegia but can walk more than six metres independently.

- Blood pressure is stable below 160/90 mm Hg.

- Enough cognition to follow commands and MiniMental State Examination score $>24$.

- Never received standard Chinese or western medicine rehabilitation treatment before the study.

- No experience of TC exercise in the past.

- Patients or their legal guardians sign the informed consents.

\section{Exclusion criteria}

- Patients who meet the diagnostic criteria of AIS but are treated by thrombolysis or surgery.

- Younger than 30 years old or older than 80 years old; or aged 30-80 years old, but left-handed habit.

- The course of this disease is more than 3 months after onset.

- Although the onset of this disease resulted in dyskinesia, the muscle strength is less than III grade (excluding III grade).

- Patients with joint lesions that affect the motor function of the limbs.

- Patients with skin or muscle lesions of the extremities that affect surface electromyography (sEMG) examination.

- Patients with severe dysfunction of heart, lung, liver, kidney and other important organs and blood system.

- Patients with moderate and severe cognitive comprehension or visual impairment, unable to complete rehabilitation treatment and examination.

- Women during pregnancy or breast feeding.

- Patients who are participating in other clinical studies.

Participants withdrawal and dropout criteria

- Patients who require to quit initiatively.

- Patients with serious cardiovascular and cerebrovascular diseases and other systemic complications or serious adverse events, such as deterioration of the disease, requiring urgent measures.

- Patients who do not receive rehabilitation treatment as prescribed.

- Patients who do not complete the information recording as required and affect the outcome assessment.

- Patients who lost to follow-up.

\section{Participants rejection criteria}

- Patients who do not meet the inclusion criteria but is strayed.

- Patients who have been randomised but have not received rehabilitation treatment. 
Table 1 Schedule of enrolment, intervention and assessments

\section{Study period}

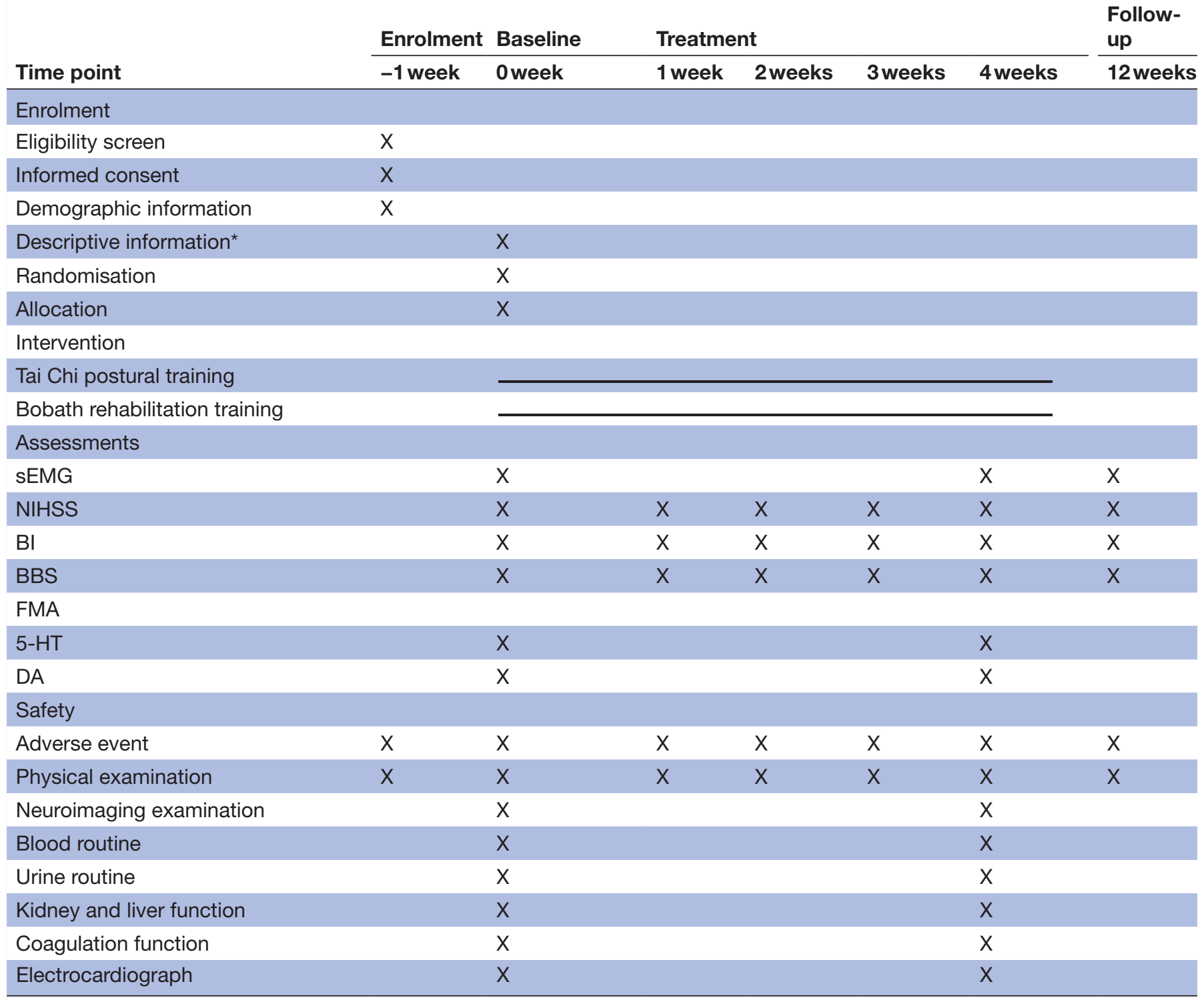

${ }^{*}$ Descriptive information includes the personal general data, vital signs, clinical symptoms and signs, history of present illness, medical history, medication history and neurological examination.

BBS, Berg Balance Scale; BI, Barthel Index; DA, dopamine; FMA, Fugl-Meyer Assessment; 5-HT, 5-hydroxytryptamine; NIHSS, National Institutes of Health Stroke Scale; sEMG, surface electromyography.

\section{Randomisation}

A total of 120 eligible AIS patients will be randomly assigned into the experimental group or the control group (60 cases per group) in a 1:1 ratio with central randomisation method. The information of each selected participant will be uploaded to the electronic data capture (EDC) system of a third-party evaluation centre in real time. Then participants will be automatically randomised through the system network. The thirdparty evaluation centre is independent of the study and its EDC system passes the international certification. Four clinical research coordinators at three trial sites will be responsible for recruiting patients, using the EDC, and requesting randomisation.

\section{Blinding}

Due to the significant difference between the TPT and the Bobath rehabilitation training, masking the therapists is difficult. But patients, clinical research coordinators, outcome assessors and statisticians who conduct the final statistical analyses will be blinded to the group allocation. The bottom of the blind will not be revealed until the statistical analyses are completed. The EDC system and training of all researchers prior to the start of the trial will ensure the successful implementation of the blinding method.

\section{Baseline assessment}

Descriptive information will be collected at baseline. Descriptive information includes the patient's personal 
general data, vital signs, clinical symptoms and signs, history of present illness, past medical history, medication history and neurological examination. At the same time, patients also need to receive head CT or MRI neuroimaging examination, blood routine, urine routine, liver and kidney function and other laboratory tests. The resulting data will be used to describe and compare the baseline characteristics of the two groups. In addition, the first sEMG data collection, the first clinical scales assessment and the first peripheral blood sample collection will be completed at baseline.

\section{Intervention}

All treatment will be implemented by the licensed rehabilitation therapists with at least 3 years of treatment experience. Each training session will last $40 \mathrm{~min}$ and will be implemented once a day and five times per week (from Monday to Friday) in a duration of 4 weeks. After each treatment, the corresponding therapist is required to sign for reference. Patients will be permitted to insist to the former treatment which benefit to their condition. Other treatment that may affect the patient's motor function will be banned, such as oral muscle relaxants, surgery and so forth.

\section{The experience group}

Patients in the control group received TPT. We selected six basic movements in the TC routine, namely, 'Starting Posture', 'Part the Wild Horse's Mane', 'Cloud Hands', 'Brush Knee and Twist Step', 'Forearm Rollings on Both Sides' and 'Closing Form', as the basic movements for patients to practice. The standard movements refer to the 24 simplified TC popularised by General Administration of Sport of China (http://www.sport.gov.cn/). In the actual training process, accuracy and coherence of postural routines will not be emphasised. Meanwhile, we will reduce the difficulty of reaching the standard of the postures appropriately according to the actual exercise ability of patients.

The upper limb training content required to complete by each basic movement is shown in table 2 . From easy to difficult, these six movements basically cover the trajectory of upper limb in daily life. Postural training also involves wrist and finger movement, which can achieve a more comprehensive upper limb rehabilitation training for patients. At the same time, the requirements of the six movements for the lower limbs include the single support movement moving forward, backward, left and right from the middle step, single limb weight training, hip, knee and ankle joint movement training, lumbar dorsal and lower limb muscle group control training, standing, and walking balance training and other rehabilitation training programmes. Depending on the patient's ability, the patient with poor muscle strength or high degree of spasm will be assisted by his or her therapist.

At the beginning of the training, the decomposition posture training of the limbs of the healthy and affected sides will be identical. With the improvement of the

\begin{tabular}{|c|c|}
\hline $\begin{array}{l}\text { Name of the basic } \\
\text { movement }\end{array}$ & The upper limb training content \\
\hline Starting posture & 1. Flex-stretch \\
\hline $\begin{array}{l}\text { Part the wild horse's } \\
\text { mane }\end{array}$ & $\begin{array}{l}\text { 1. Stretch-adduction } \\
\text { 2. Flex-adduction }\end{array}$ \\
\hline Cloud hands & $\begin{array}{l}\text { 1. Flex-adduction-extorsion } \\
\text { 2. Flex-stretch-abduction }\end{array}$ \\
\hline $\begin{array}{l}\text { Brush knee and twist } \\
\text { step }\end{array}$ & $\begin{array}{l}\text { 1. Flex-abduction-extorsion- } \\
\text { adduction } \\
\text { 2. Flex-adduction-intorsion-stretch }\end{array}$ \\
\hline $\begin{array}{l}\text { Forearm rollings on } \\
\text { both sides }\end{array}$ & $\begin{array}{l}\text { 1. Flex-abduction-extorsion-flex- } \\
\text { adduction-intorsion } \\
\text { 2. Flex-adduction-intorsion-flex- } \\
\text { abduction-extorsion }\end{array}$ \\
\hline Closing form & 1. Stretch-flex \\
\hline
\end{tabular}

patients' active exercise ability, different posture training should be completed on the left and right sides at the same time. Each of the six basic movements requires a full postural interaction with a combination of the healthy and the affected side. The enrolled AIS patients do not need to practice all six basic movements, but repeated exercises selectively according to their respective dysfunction points, and gradually advanced exercises according to the difficulty of the movements.

To ensure the quality of the research, the therapists in the experimental group will receive instruction and training of TPT techniques. The content is divided into three parts, including the theoretical explanation of six basic movements, the demonstration of posture control techniques and the practice of actual operation. After the training, an assessment will be organised. Only the therapists who pass the examination can get the certificates. The intervention programme of the experimental group must be conducted by the certified rehabilitation therapists at all participating centres. Video recording is required during the treatment.

\section{The control group}

Patients in the control group will receive standard modern Bobath rehabilitation training. According to the 'Chinese Guidelines for Stroke Rehabilitation' (2011 version), ${ }^{13}$ the main contents of training include good limb position, muscle strength and joint activity training, knee-ankle joint control training, weight loss gait training and so on. The above rehabilitation training content can be selectively carried out to enhance training effect. All trainings focus on changing the patient's abnormal posture and abnormal movement patterns.

To ensure the quality of the research, the therapists in the control group will participate in the internationally certified Bobath technical training and obtain the certificates after the examination. The intervention programme 
of the control group must be conducted by the certified rehabilitation therapists at all participating centres.

\section{Follow-up}

After the 4-week treatment, the participants will enter a 3-month follow-up period. During the follow-up period, all patients will be encouraged to continue community or family rehabilitation training. At the same time, patients in the experimental group will be encouraged to continue TPT. The training frequency is required to be more than three times per week. Patients in both groups will be given rehabilitation training diaries, and need to record the content and duration of rehabilitation training every day with the assistance of family members. Researchers will call the patients every 2 weeks to urge their rehabilitation training and keep a record of follow-up time. During the follow-up, the patients' combined medication status will be recorded in detail for further correlation analysis.

At the end of the 3-month follow-up period, all patients will be required to return to the hospital for SEMG examination and clinical scales assessment.

\section{Outcome measures}

In this study, the primary outcome and secondary outcomes will be measured at baseline, the end of the 4-week treatment and the end of the 3-month follow-up. Peripheral blood sample will be collected at baseline and the end of 4-week treatment. All outcome assessments will be independently performed by experienced and blinded assessors, who are also professional rehabilitation physicians.

\section{Primary outcome}

\section{Root Mean Square}

Since muscle strength is a major indicator of motor function, root mean square (RMS) generating from FlexComp sEMG system (Thought Technology, Canada) will be selected as the primary outcome. Such timedomain parameter reflects the effective value of the muscle discharge. Its peak value indicates the amplitude or contraction intensity, which mainly depends on the internal relationship between the muscle load factor and the physiological (also biochemical) processes of the muscle itself. RMS, an objective data indicator, is more sensitive and reliable in reflecting the changes of muscle strength than clinical muscle strength examination, which is only divided into $0-5$ grades.

Electromyographic information will be collected from 11 muscles, including biceps brachii, triceps brachii, flexor carpi ulnaris, extensor digitorum, deltoid, quadriceps femoris, hamstring tendon, tibialis anterior, gastrocnemius, peroneus longus and erector spinae. Bilateral limb muscles collection will be conducted, a total of 22 muscles. Data from the healthy limbs will be used as a normal control.

The acquisition of single muscle EMG information will begin in the patient's resting state (Only if the heart rate ranging from 60 to 100 beats per minute will be permitted). When connected to the sEMG system, the electrode plate will be pasted in the muscle abdomen according to the highest EMG signal point which will be automatically recognised by the sEMG system. When the EMG signal is stable, the assessors will ask the patient to exercise according to the time sequence of forced contraction and relaxation under the system prompt. After the collection of isometric movement, the data of muscle dynamic isotonic movement will be collected. The above tests will be performed again after an 1 min rest. With a total of three cycles, the average value of RMS will be taken.

\section{Secondary outcomes \\ Integrated EMG}

Integrated EMG (IEMG) is the total amount of electrical discharge of the motor units participating in muscle activities over a period of time, which is positively correlated with muscular tension. Under the premise of time invariable, IEMG can reflect the number of motor units and the discharge level of each motor unit. The value decreases linearly with the load duration. Considering the importance of muscular tension in the rehabilitation of motor function, IEMG will be selected to assess the state of muscular tension.

\section{Average EMG}

Similar to RMS, average EMG (AEMG) is also a characteristic indicator of signal amplitude variation, which is the average amplitude of instantaneous EMG over a period of time. AEMG can reflect the amount of motor unit activation, the type of motor unit participating in the activity, and the degree of synchronisation during muscle activity. Therefore, AEMG is also an important objective data indicator to express muscle strength level.

\section{Mean Power Frequency and Median Frequency}

Mean power frequency (MPF) and median frequency (MF) are both frequency-domain parameters that reflect the frequency characteristics of EMG signals. The former is related to the proportion of fast and slow muscle fibres in muscle tissue. The latter is related to the conduction velocity of action potential, the type of moving unit and the degree of synchronisation. These two parameters can be used to measure fatigue during muscle activity.

\section{Clinical Scales Assessment}

Fugl-Meyer Assessment (FMA), Berg Balance Scale (BBS), Barthel Index (BI) and National Institutes of Health Stroke Scale (NIHSS) will be selected to evaluate the rehabilitation effect of motor function in AIS patients. FMA will be used to assess reflexes, spasms, and motor coordination in patients. BBS will be used to assess balance ability of patients. BI will be used to assess the patients' daily living ability. NIHSS will be used to assess neurological function impairment in patients. 


\section{Safety outcomes}

Routine blood and urine tests, coagulation function test, liver and kidney function tests and ECG test will be performed before and after the intervention. The test reports and the patient's vital signs will be used as the safety outcomes.

\section{Collection, preservation and determination of peripheral blood sample}

Peripheral blood samples will be collected at baseline and at the end of 4-week treatment. Patients will be required to refrain from eating and drinking after 12:00 pm the night before blood collection, followed by venous blood collection in the morning, with a single collection of $4 \mathrm{~mL}$. The collected blood samples will be preserved in the clinical biobank for classification and storage at low temperature, and will be detected within 1 week. Two monoamine neurotransmitters, 5-hydroxytryptamine (5-HT) and dopamine (DA) will be the main determination objects.

\section{Data management and monitoring}

Before the trial, an independent data safety and monitoring committee (DSMC) will be set up for the data management and monitoring. DSMC is composed of experts from third party evaluation centres and has no conflict of interest. Researchers who are responsible for data collection and entry will be trained by DSMC to ensure the accuracy, completeness and consistency of the data. During the trial, the inspectors from DSMC will regularly monitor the correct execution of the study protocol and the correct completion of the electronic case report form (eCRF).

The EDC system will be used in this study, and all participants' visit data will be directly input into the eCRF through the network user interface in all trial centres. The data of each eCRF will be logged twice by two independent operators. The inspectors from DSMC will regularly check and verify the consistency of the original data with the input data. The checked and qualified eCRF will be signed and then sent to the data management centre of EDC system in time. The third-party data manager will establish the corresponding database according to the contents of eCRF and the requirements of statistical analysis. After entry, verification and confirmation of all correct data, the chief researcher and statistical analysts will lock the database. The locked data file cannot be modified.

\section{Quality control}

A dedicated quality control group will be established for training four clinical research coordinators. The training includes patient recruitment, disease assessment and data collection, which will minimise selection bias. In the process of data collection, patients' combined medication and non-intervention treatment will be strictly noted. The influence of the above-mentioned interfering factors will be excluded in the statistical analysis.
The quality control group will also be answerable for the long-term communication and health education of patients. They will inform patients of the potential benefits and risks of participating in the research. If an adverse event occurs during the trial, the patient will receive consultation and free treatment from professional physicians, regardless of whether it is related to the research. The above measures will help to improve compliance of patients and reduce the lost rate of follow-up.

\section{Statistical methods \\ Sample size}

The sample size calculation was based on the parameters generated from the sEMG. According to a previous small simple size study, ${ }^{29} 4$ weeks after TC intervention, an increase in muscle strength of $12.86 \% \pm 1.34 \%$ in patients treated with TC training vs $3.18 \% \pm 23.45 \%$ in patients treated with routine rehabilitation therapy. We assume that the non-inferiority limit is $25 \%$, with a type I error of $2.5 \%(\alpha=0.025)$ and $80 \%$ power $(\beta=0.20)$, so the estimated required sample size is 50 participants per group based on the formula:

$$
n=\frac{\left(Z_{1-\alpha}+Z_{1-\beta}\right)^{2}\left[p_{1}\left(1-p_{1}\right)+p_{2}\left(1-p_{2}\right)\right]}{(\varepsilon-\delta)^{2}}
$$

Considering a $20 \%$ drop-out rate during the study, 60 patients will be enrolled in each group and the total sample size will be 120 .

\section{Data sets}

In this study, three data sets will be established and analysed:

- Full analysis set (FAS): a collection of eligible and drop-out cases, but excluding rejection cases. The criteria are that the participants have been randomised and have had at least one treatment record. We will conduct intention-to-treat analysis through the FAS, which is mainly used for baseline comparability assessment and efficacy evaluation. Missing values will be filled in by the last observed carry-over.

- Per-protocol set (PPS): a collection of cases who met the inclusion criteria and completed all treatment measures. We will conduct PP analysis through the PPS, which is mainly used for efficacy evaluation.

- Safety Set (SS): a collection of cases who received at least one treatment and had a documented safety profile. Safety missing values cannot be carry-over. The SS will be used for safety analysis.

\section{Statistical analysis}

A statistician who is independent from the trial will be responsible for the statistical analysis. The SPSS V.25.0 (IBM) for Windows will be used. Statistical significance tests will be conducted on both sides, and $\mathrm{p}<0.05$ will be considered statistically significant. Descriptive statistical analysis of quantitative data will be conducted by means of mean, SD, number of cases, minimum, maximum, 95\% CIs or median, upper quartile (Q1), lower quartile (Q3). Comparisons will 
be made between and within groups as required. For intergroup comparison, if the data of the two groups conform to normal distribution and homogeneity of variance, independent sample t-test will be adopted; otherwise, Wilcoxon rank sum test will be adopted. For intra-group comparison, if the difference values conform to normal distribution, the paired sample t-test is adopted; otherwise, the sign rank sum test is adopted. The differences between the central effector and other confounding factors will be compared by using covariance analysis. Descriptive statistical analysis of qualitative data will be conducted by frequency and composition ratio. Comparisons will also be made between and within groups. $\mathrm{X}^{2}$ or Fisher's exact test will be used for intergroup comparison, and Wilcoxon rank-sum test will be used for rank data. McNemar's test will be used for intra-group comparison. CochranMantel-Haenszel test will be applied if the central effect is considered.

\section{Patient and public involvement}

Patients and/or the public were not involved in the design, or conduct, or reporting, or dissemination plans of our research.

\section{ETHICS AND DISSEMINATION \\ Ethics}

The study has been approved by Ethics Committee of Dongzhimen Hospital Affiliated to Beijing University of Chinese Medicine, being granted approval numbers DZMEC-KY-2020-22. We will follow the Declaration of Helsinki 2013 and will report the protocol according to the Standard Protocol Items: Recommendations for Interventional Trials 2013 Statement.

\section{Safety}

Any adverse events occurring during the trial will be recorded in detail regardless of whether they are related to the research. Once an adverse event occurs, the participant will be required to truthfully report changes in their condition before and after treatment. At the same time, the researchers will immediately take corresponding treatment measures until the participant' condition is stable. Within the next 24 hours, details of the adverse events will be reported to the hospital ethics committee, including the time of occurrence, clinical symptoms and signs, severity, duration, physical and chemical examination indexes, treatment methods and results, etc. The ethics committee will assess the correlation between the adverse events and the intervention, and will make a final decision on whether the participant will continue to participate in the trial. During the period of adverse events, the researchers will further record changes in the adverse events reported by the participant (such as whether the adverse event subsided or if it still exists, its severity and frequency), and determine whether the adverse events occurred are related to the trial.

\section{Dissemination}

The research results will be disseminated through (open access) peer-reviewed publications and presentations at conferences.

\section{DISCUSSION}

Although preliminary progress has been made in the clinical practice of TC for dyskinesia rehabilitation, these researches are mainly based on integral TC routine. Meanwhile, as a complementary and alternative therapy, TC has its limitations and still hardly be applied for AIS patients with severe dyskinesia. In this trial, the standard practice postures of TC are simplified and decomposed according to the actual situation of the patients. 'Cloud Hands', for instance, can be simplified and decomposed into 'The unilateral upper limb movement of the Cloud Hands', 'The two-sided upper limb movement of the Cloud Hands', 'The unilateral upper limbs combined with trunk rotation movement of the Cloud Hands', 'The two-sided upper limbs combined with trunk rotation movement of the Cloud Hands', 'Standard Cloud Hands movement', 'Anti-resistance Cloud Hands movement'. Regardless of whether the patient is bedridden or able to stand or walk, the above training movements can meet the rehabilitation training requirements of different degrees of dyskinesia. The rehabilitation method performing from healthy side to the affected side is used in the specific training process, so that the affected side gradually adapt to the weight training. For the patients with weak balance and coordination function, the static training of fixed motion will be implemented. The dynamic postural training will be started based on strengthening static motion. Through simplifying and decomposing standard practice postures of TC, combined with the assistance of the therapist or moderate antiresistance postural training, TPT can be applied to convalescent ischaemic stroke patients in different Brunnstrom stages. In actual training, TPT will be selected according to the patients' changing characteristics of mechanics. The key point lies in improving muscle strength and inhibiting muscle spasm, which will further induce the corresponding cortical neural reflex development.

Studies on the application of TC for dyskinesia are short of unified standard for the efficacy evaluation, among which the subjective clinical scale assessment is the most widely used. sEMG, which reflects the state of neuromuscular activity by electromyographic signals during rest or movement, has been widely used in the field of rehabilitation medicine. ${ }^{30}$ Based on the characteristic description of limb muscle strength, muscular tension and muscle group coordination, sEMG can provide more objective data supporting for the evaluation of limb motor function. ${ }^{31}$ In a previous study, researchers used sEMG to test healthy participants who are practicing TC. The results 
indicated that the participants got significant improvement in muscle group stability, coordination and fatigue resistance. ${ }^{32}$ A recent study had also confirmed that sEMG can objectively evaluate the upper limb motor function and abnormal gait of AIS patients. ${ }^{33}$ Therefore, sEMG will be introduced in this study to provide objective parameters for the efficacy evaluation of TPT.

In addition to verifying clinical efficacy, another important objective of this study is to explore the central-peripheral neurotransmitter mechanism of TPT. Changes of central excitatory or inhibitory functions have important effects on motor function, among which the regulation of monoamine neurotransmitters is the most critical. $^{34-37}$ Several studies have shown that change of monoamine neurotransmitters level such as 5-HT and DA within the brain was closely related to central fatigue. ${ }^{38-41}$ One of the results showed that an increase in central ratio of 5-HT to DA was associated with feelings of tiredness and lethargy. ${ }^{38}$ The interaction between 5-HT and DA was also reported to play a regulative role in the development of central fatigue, ${ }^{3740}$ among which the 5 -HT played the dominant role in the dynamic changes. ${ }^{36} 41 \mathrm{~A}$ small sample trial about TC also showed significant differences in 5-HT and DA levels before and after the TC exercise, which suggested that the TC exercise was closely related to change of monoamine neurotransmitters level in the blood. $^{42}$

Some limitations are inevitable in this study. First, although the condition of AIS patients in convalescence is more stable than that in the acute phase, relapse or severe complications may lead to a high drop-out rate, which will affect the final statistical analysis. Second, because of the characters of the intervention, the therapists are not blind. Third, the limitation of sample size and region may affect the further popularisation of the research results. Despite facing above challenges, we will strive to standardise the steps in the trial. We hope that the results of this trial will provide more reliable evidence for the application of TPT in the convalescence of AIS, and attempt to elucidate the efficacy mechanisms.

\section{Trial status}

At the time of submission, the trial is currently in the recruitment phase. This trial started on 1 June 2020 and will end on 31 December 2022.

Acknowledgements The group thanks all patients who participated in this trial for their cooperation. The authors also would like to express their gratitude to the anonymous reviewers for their excellent work and constructive criticisms.

Contributors CJ and KL are joint first authors of this manuscript, contributing equally to the drafting of the manuscript. ZL conceived and designed the study protocol. YS, JW, YiL and YuL helped develop the study measures and data collection. YZ, LZ, ZZ and MZ provided stroke rehabilitation and clinical scales evaluation expertise. All authors read the final manuscript and approved its submission.

Funding This work was supported by grants from University-level Key Project of Beijing University of Chinese Medicine (No. 2020-JYB-ZDGG-112) and National Natural Science Foundation of China (81673894).

Disclaimer The fund sources had no role in the design of the study, the data collection, analysis, and interpretation of data, or writing the manuscript.
Competing interests None declared.

Patient and public involvement Patients and/or the public were not involved in the design, or conduct, or reporting, or dissemination plans of this research.

Patient consent for publication Not required.

Provenance and peer review Not commissioned; externally peer reviewed.

Open access This is an open access article distributed in accordance with the Creative Commons Attribution Non Commercial (CC BY-NC 4.0) license, which permits others to distribute, remix, adapt, build upon this work non-commercially, and license their derivative works on different terms, provided the original work is properly cited, appropriate credit is given, any changes made indicated, and the use is non-commercial. See: http://creativecommons.org/licenses/by-nc/4.0/.

\section{ORCID iDs}

Chengyang Jing http://orcid.org/0000-0002-6035-2855

Kuangshi Li http://orcid.org/0000-0001-7998-269X

Zongheng Li http://orcid.org/0000-0001-8906-2563

Yiting Sun http://orcid.org/0000-0002-9615-8798

Jiabao Wu http://orcid.org/0000-0002-8648-7360

Yingjie Li http://orcid.org/0000-0001-9503-8172

Yuyue Li http://orcid.org/0000-0002-5416-038X

Li Zhou http://orcid.org/0000-0002-2739-1211

Zhe Zhang http://orcid.org/0000-0002-4249-1966

Mingzhi Zhao http://orcid.org/0000-0003-4535-7620

Yong Zhang http://orcid.org/0000-0001-7985-8031

\section{REFERENCES}

1 Feigin VL, Forouzanfar MH, Krishnamurthi R, et al. Global and regional burden of stroke during 1990-2010: findings from the global burden of disease study 2010. Lancet 2014;383:245-55.

2 Liu L, Wang D, Wong KSL, et al. Stroke and stroke care in China: huge burden, significant workload, and a national priority. Stroke 2011;42:3651-4.

3 Wang W, Jiang B, Sun $\mathrm{H}$, et al. Prevalence, Incidence, and Mortality of Stroke in China: Results from a Nationwide Population-Based Survey of 480687 Adults. Circulation 2017;135:759-71.

4 Wang D, Liu J, Liu M, et al. Patterns of stroke between university hospitals and nonuniversity hospitals in mainland China: prospective multicenter hospital-based registry study. World Neurosurg 2017;98:258-65.

5 Wang Z, Li J, Wang C, et al. Gender differences in 1-year clinical characteristics and outcomes after stroke: results from the China National Stroke Registry. PLoS One 2013;8:e56459.

6 Zi-long H, Ming L, Wei LI. Basic characteristics and functional outcomes of 3123 consecutive patients in Chengdu stroke registry. Chinese J Neurol 2011;44:826-31.

7 Wang Y-J, Zhang S-M, Zhang L, et al. Chinese guidelines for the secondary prevention of ischemic stroke and transient ischemic attack 2010. CNS Neurosci Ther 2012;18:93-101.

8 Karasu AU, Batur EB, Karataș GK. Effectiveness of Wii-based rehabilitation in stroke: a randomized controlled study. J Rehabil Med 2018;50:406-12.

9 Așkın A, Atar E, Koçyiğit $\mathrm{H}$, et al. Effects of Kinect-based virtual reality game training on upper extremity motor recovery in chronic stroke. Somatosens Mot Res 2018;35:25-32.

10 Jie L-J, Kleynen M, Meijer K, et al. The effects of implicit and explicit motor learning in gait rehabilitation of people after stroke: protocol for a randomized controlled trial. JMIR Res Protoc 2018;7:e142.

11 Steenbergen B, van der Kamp J, Verneau M, et al. Implicit and explicit learning: applications from basic research to sports for individuals with impaired movement dynamics. Disabil Rehabil 2010;32:1509-16.

12 Tollár J, Nagy F, Csutorás B, et al. High frequency and intensity rehabilitation in 641 subacute ischemic stroke patients. Arch Phys Med Rehabil 2021;102:9-18.

13 Society CN, Society CS. Chinese guidelines for the treatment and rehabilitation of the stroke (2011 full version). Chinese J Rehab Theory Pract 2012;18:301-18.

14 Levin MF, Panturin E. Sensorimotor integration for functional recovery and the Bobath approach. Motor Control 2011;15:285-301.

15 Brock K, Haase G, Rothacher G, et al. Does physiotherapy based on the Bobath concept, in conjunction with a task practice, achieve greater improvement in walking ability in people with stroke compared to physiotherapy focused on structured task 
practice alone?: A pilot randomized controlled trial. Clin Rehabil 2011:25:903-12.

16 Tang Q, Tan L, Li B, et al. Early sitting, standing, and walking in conjunction with contemporary Bobath approach for stroke patients with severe motor deficit. Top Stroke Rehabil 2014;21:120-7.

17 Kollen BJ, Lennon S, Lyons B, et al. The effectiveness of the Bobath concept in stroke rehabilitation: what is the evidence? Stroke 2009;40:E89-97.

18 Taylor-Piliae RE, Haskell WL. Tai chi exercise and stroke rehabilitation. Top Stroke Rehabil 2007:14:9-22.

19 Ding M. Tai chi for stroke rehabilitation: a focused review. Am J Phys Med Rehabil 2012;91:1091-6.

20 Lu Y, Li J, Xiao B, et al. Clinical research situation and thinking of Taijiquan in foreign countries. Chinese $J$ Int Tradit Western Med 2013;33:1717-21.

21 Zheng G, Li S, Huang M, et al. The effect of tai chi training on cardiorespiratory fitness in healthy adults: a systematic review and meta-analysis. PLoS One 2015;10:e0117360.

22 Zheng G, Lan X, Li M, et al. The effectiveness of tai chi on the physical and psychological well-being of college students: a study protocol for a randomized controlled trial. Trials 2014;15:129.

23 Lan C, Chen S-Y, Lai J-S, et al. Tai chi chuan in medicine and health promotion. Evid Based Compl Alternat Med 2013;2013:1-17.

24 Wang $\mathrm{C}$, Schmid $\mathrm{CH}$, Rones $\mathrm{R}$, et al. A randomized trial of tai chi for fibromyalgia. N Engl J Med 2010;363:743-54.

25 Li F, Harmer P, Fitzgerald K, et al. Tai chi and postural stability in patients with Parkinson's disease. N Engl J Med 2012;366:511-9.

26 Zheng G, Huang M, Liu F, et al. Tai chi chuan for the primary prevention of stroke in middle-aged and elderly adults: a systematic review. Evid Based Complement Alternat Med 2015;2015:1-18.

27 Zhou Q, Xu J, Hu A. Observation on recover of cerebral infraction patients by Taijiquan training. Chinese $J$ Pract Nervous Dis 2010;13:20-2.

28 Society CN, Society CS. Chinese guidelines for diagnosis and treatment of acute ischemic stroke 2018. Chinese $J$ Neurol 2018;51:666-82.

29 YANG H, LIU X. Effects of Taiji Quan and Baduanjin on motor function of lower limbs for stroke patients using surface electromyography. Chinese J Rehab Theory Pract 2019;25:101-6.
30 Gallina A, Pollock CL, Vieira TM, et al. Between-day reliability of triceps surae responses to standing perturbations in people post-stroke and healthy controls: a high-density surface EMG investigation. Gait Posture 2016:44:103-9.

$31 \mathrm{Hu}$ X, Suresh AK, Rymer WZ, et al. Altered motor unit discharge patterns in paretic muscles of stroke survivors assessed using surface electromyography. J Neural Eng 2016;13:046025.

32 Wang R, Yang Q. Electromyogram changes in practicing Taijiquan of different schools. J Beijing University Phys Educat 1996;04:39-42.

33 Fang L, Jie-jiao Z. Application of surface electromyography in abnormal gait analysis for stroke (review). Chinese $J$ Rehab Theory Pract 2016;22:1159-62.

34 Shen K-Z, Johnson SW. 5-HT inhibits synaptic transmission in rat subthalamic nucleus neurons in vitro. Neuroscience 2008:151:1029-33.

35 Xu C-X, Liu H-T, Wang J. Changes of 5-hydroxytryptamine and tryptophan hydroxylase expression in the ventral horn of spinal cord. Neurosci Bull 2008;24:29-33.

36 Yang D-S, Liu X-L, Qiao D-C. [Dynamic changes of 5-HT, DA and their metabolin in rat striatum during exhaustive exercise and recovery]. Zhongguo Ying Yong Sheng Li Xue Za Zhi 2011;27:432-6.

$37 \mathrm{Hu}$ Y, Liu X, Qiao D. Increased extracellular dopamine and 5-hydroxytryptamine levels contribute to enhanced subthalamic nucleus neural activity during exhausting exercise. Biol Sport 2015;32:187-92.

38 Meeusen R, Watson P, Hasegawa $\mathrm{H}$, et al. Central fatigue: the serotonin hypothesis and beyond. Sports Med 2006;36:881-909.

39 Leite LHR, Rodrigues AG, Soares DD, et al. Central fatigue induced by losartan involves brain serotonin and dopamine content. Med Sci Sports Exerc 2010;42:1469-76.

40 Wright HE, Selkirk GA, Rhind SG, et al. Peripheral markers of central fatigue in trained and untrained during uncompensable heat stress. Eur J Appl Physiol 2012;112:1047-57.

41 Kavanagh JJ, McFarland AJ, Taylor JL. Enhanced availability of serotonin increases activation of unfatigued muscle but exacerbates central fatigue during prolonged sustained contractions. J Physiol 2019;597:319-32.

42 Xiang-xian C. The effect of Taijiquan on the change of content ingredients of monoamine class neurotransmitter and the curing effect. J Beijing University Phys Educat 2003;05:633-4. 